\title{
群落结构复杂性的测度方法研究进展
}

\author{
金 森 \\ (东北林业大学林学院，哈尔滨 150040)
}

\begin{abstract}
摘 要 该文对群落结构复杂性的测度方法的研究进展状况进行了综述。根据测度方法建立的方法基础 将现有 的方法分成 3 类 基于多样性的复杂性测度、基于计算复杂性的测度和基于几何学特征的复杂性测度。对每类测 度方法进行了介绍, 对其优缺点进行了评述。同时提出了未来研究中应给予重视的问题。结果表明 现有群落结 构复杂性的测度方法普遍存在区分能力差的问题，对于基于多样性的结构复杂性测度，目前还缺乏确定各测度属 性权重的客观方法 现有的一些基于计算复杂性的结构测度与多样性指标关系过于密切, 还不完善, 同时其生态学 的意义还不明确，而另一些计算复杂性指标还缺乏实际检验。今后，如何建立既具有区分力、又与多样性在概念和 数值上都有一定区别的群落结构的计算复杂性的测度方法、如何科学合理地确定复杂性测度中的属性权重以及如 何建立结构复杂性的测度和功能过程之间的联系等都是需要深入和系统研究的。由于方法的相似性, 有关群落结 构复杂性的测度方法也可以应用到其它尺度上的结构复杂性的研究中。
\end{abstract}

关键词 复杂性 结构 计算复杂性 测度 科尔莫哥洛夫复杂性 霍夫曼编码 生态复杂性

\section{A REVIEW ON METHODS FOR MEASURING COMMUNITY STRUCTURAL COMPLEXITY}

\author{
JIN Sen \\ ( College of Forest Science, Northeast Forestry University , Harbin 150040 , China)
}

\begin{abstract}
Ecological complexity has received increasing attention in recent years. Structural complexity is one of the most important parts of ecological complexity. This paper reviews the literature on the development of concepts and measures of community structure. Findings indicate that currently used methods can be classified into three groups : measures based on biodiversity, algorithmic complexity and geometrical properties. The many measures based on biodiversity are commonly used. The algorithmic method is new and has not been widely used. It represents the complexity of community structure by the difference of mean length of Huffman code of community attributes and 12th order of Rényi entropy. The methods based on geometrical properties are also commonly used, employing fractal dimension as the most important index. A common problem for all these methods is the difficulty of comparing community complexity with different complexity measures. Also, these methods weakly discriminate among different structures. For the methods based on biodiversity , it is difficult to objectively determine weights of the attributes used. For the algorithmic complexity based methods, the ecological meanings of the measures are still uncertain. Some of them are closely correlated with biodiversity indices , and the others need field testing. Future studies should focus on the following:1) Determine algorithmic measures with high discriminant ability that differ from biodiversity indices both in concept and values. Because many algorithmic measures have been widely used in fields other than ecology , interesting results could be generated when applying these measures to description of complexity of community structure. 2) Objectively determine weights of attributes with ecological meaning used in complexity measures, a problem that exists in biodiversity and algorithmic measures that use multiple attributes. 3) Connect measures of structural complexity with functions and processes, a critical goal in the study of complexity of community structure.
\end{abstract}

Key words Complexity, Structure, Algorithmic complexity, Measure, Kolmogorov complexity, Huffman code , Ecological complexity

复杂性 (Complexity) 问题是近年来研究热点之 一(马飞等,2003; 吴彤,2004)，随着科技的发展，对
复杂系统与复杂性有了新的认识,引起了诸多领域 科学家的广泛关注( Rind ,1999; 蔡立羽等, 2000; Hao 
et al. 2002; ;马飞等,2003; 赵文杰和刘兆理, 2003; Boero et al .2004; Vogradov et al., 2004 ;佟春生等， 2005 薛小杰和谭克龙, 2005)。生态学更是如此(张 知涁等, 1998; Marquet,2000;王莉等,2002)。2004 年 创刊的 Ecological Complexity”杂志，标志着生态复杂 性的研究进入了一个崭新阶段。过去生态复杂性研 究主要集中在群落或生态系统复杂性与稳定性之间 的关系上。近年来, 利用复杂性科学的原理和方法 研究进化和生态学问题的工作日益增多, 涉及到种 群、群落、生态系统乃至景观等不同层次上的结构和 功能 ( Anand \& Orlóci ,1996 ;Metzger \& Muller ,1996; Parrish \& Edelstein-Keshet ,1999; Moser et al. ,2002 ; Kondoh , 2003 ; Lindsey , 2004 ; Bolliger et al . , 2005 ; Hoegh-Guldberg , 2006)。

这些研究可分成两大类 :一类是生态复杂性的 测度问题，即解决各个生态等级上的结构、组成、格 局以及它们在时间上的状态变化即动态的复杂性描 述问题。另一类是功能复杂性问题, 即用复杂性的 方法研究形成这些复杂结构、格局背后的功能、过程 规律 (Bascompte \& Solé , 1995 ;Storch \& Gaston , 2004 ; Maurer, 2005)。目前, 关于后者的研究似乎远多于 前者取得了很多进展，如利用模型和计算机方法， 揭示了许多复杂格局往往是由简单规律形成的 (Bascompte \& Solé , 1995 ;郝柏林,2001;Fleurant et al . , 2004 ;Keränen , 2004 ;Bolliger et al . 2005;Dupuy et al . , 2005 ;Green \& Sadedin ,2005)。相比之下, 复 杂性的测度研究相对滞后, 主要原因是傅杂性的测 度取决于复杂性的定义和问题自身的难度。复杂性 的定义本身就相对广泛 (吴䑣,2004), 目前还没有形 成共识。而对于其中任何一种定义，建立一个合适 的测度体系本身也比较困难。尽管如此,生态学中 的结构复杂性的测度问题, 始终是生态复杂性研究 的重要理论基础。没有明确的生态结构复杂性定义 和测度方法, 其它研究的意义就会有所淡化, 不同研 究之间也无法比较。因此, 生态学中的结构复杂性 的测度研究急需加强。在生态结构复杂性的测度研 究中, 目前关于群落结构复杂性的测度研究工作开 展较多, 因此, 本文对以群落结构为对象, 对其研究 情况进行综述。由于在生态学中各层次或尺度上的 结构复杂性的描述方法具有很大的相似性, 用于描 述群落结构复杂性的方法完全可以用于分子、种群、 景观等尺度上的生态结构复杂性的描述, 事实上这 些方法也在这些领域有所应用。因此, 本文的综述 对于整个生态结构复杂性的测度研究具有一定的代
表性。

复杂性及其测度本不来自生态学, 而是从计算 机科学等其它科学中借鉴发展而来。群落结构复杂 性的测度方法也不例外。为清楚和方便起见，先简 要介绍复杂性测度的发展过程中所出现的各种概念 和测度方法 然后再介绍群落结构复杂性的主要测 度方法。

\section{1 复杂性的概念和测度}

复杂性研究自 20 世纪中叶兴起，从历史角度来 看, 贝塔朗菲创立一般系统论标志着复杂性科学的 诞生, 依据研究对象的变化, 可把复杂性科学的发展 历史大致划分为 3 个阶段:第一阶段, 研究存在;第 二阶段，研究演化，第三阶段，综合研究阶段(金吾伦 和郭元林, 2004)。在此过程中, 产生了 50 多种复杂 性的概念和相应的测度。吴粀(2004)对这些概念进 行了分类和比较研究, 将其大体区分为 3 类:计算 型、多样结构型和隐喻型。其中, 计算型复杂性是一 维序列的复杂性程度的度量, 可以包括以下 6 类: :1) 信息类 如费希尔信息、储存信息、算法信息含量等; 2)熵类 :Rényi 熵、计量熵等;3) 描述长度或距离类 : 自描述代码长度、最小描述长度等; 4 ) 容量类: 拓扑 机器容量等 ; $)$ 深度类 逻辑深度、热力学深度等 ;6) 复杂性类 :Lempel-Ziv 复杂性 (Lemper \& Ziv，1976)、 随机复杂性等。自然科学和计算机科学等工程技术 领域的复杂性多为计算型的复杂性概念。多样结构 型复杂性是纵向层次之间的复杂程度的度量, 可以 包括两类:1)多样性类 树形多样性、区别性等，2)独 立参数个数或维数参数个数或自由度或维数、分维。 隐喻型复杂性概念是前两类复杂性度量之外的更大 语境中的系统与历史、与演化、与环境的相互关系的 复杂程度的度量。包括混合、相关性、分辨力、自组 织、自组织临界性、复杂适应系统、报酬递增、路径依 赖、适切景观、涌现、生成关联、混沌边缘、自相似、模 拟退火、奇怪吸引子等。在系统科学的范式下, 以多 样性、异质性为基础的进行复杂性的研究, 把复杂性 与异质性、数量巨大、层次性、结构多样联系在一起。 这与多样结构型和隐喻型的复杂性定义有密切的联 系。

吴䑣 (2004) 认为在这 3 类复杂性概念中, 本质 基础应是计算复杂性的概念。科尔莫哥洛夫（Kolmogorov) 复杂性概念是多数计算型的复杂性的基 础。它与是否能够构造一个对象的算法及算法的计 算量大小有关。这种类型的复杂性概念描述即 计 
算型” 复杂性。该复杂性研究起源于 20 世纪 30 年 代对可计算理论及其算法的研究。科尔莫哥洛夫复 杂性概念的一般数学形式为:

$$
\begin{aligned}
& \mathrm{Ks}(x)=\min \{|p|: \mathrm{S}(p)=\mathrm{n}(x)\} \\
& \mathrm{Ks}_{\mathrm{s}}(x)=\infty \text {, 如果不存在 } p,
\end{aligned}
$$

其含义为 :对每一个 $\mathrm{D}$ 域中的对象 $x$, 我们称最小程 序 $p$ 的长度 $|p|$ 就是运用指定方法 $\mathrm{S}$ 产生的关于对 象 $x$ 的科尔莫哥洛夫复杂性。对计算机 $S$ 而言, 设 给定的符号串为 $x$ 将产生 $x$ 的程序记为 $p$ 。对一个 计算机来说, $p$ 是输入, $x$ 是输出。粗略地说, 关于 一个符号串 $x$ 的科尔莫哥洛夫复杂性, 就是产生 $x$ 的最短程序 $p$ 的长度。这与早期根据对象事件的可 预测性来定义的复杂性的定义和测度是一致的。一 个事件能够用较简单的函数来描述或用较短的代码 /符号串来刻画，表明此事件可预测性大，复杂性小。 因此, 按形成基础 现有的复杂性测度可分成科尔莫 哥洛夫类测度和非科尔莫哥洛夫测度。吴粀 (2004) 的分类中多数计算型属科尔莫哥洛夫类测度, 其它 的,包括一些计算型和多样结构型及隐喻型都属于 非科尔莫哥洛夫类测度。

\section{2 群落结构复杂性的定义及测度方法}

现有的生态复杂性研究可包括上述 3 种类型的 复杂性概念, 以系统科学为基础的多样结构型和隐 喻型的复杂性定义所占的比例更大 (张知彬等, 1998 ;王莉等 ,2002) , 主要体现在复杂生态系统的功 能研究上。对于群落结构复杂性的定义, 则主要是 计算型测度。根据测度方法形成的基础, 作者将群 落结构复杂性的测度分成 3 类:1) 基于生物多样性 的复杂性测度方法 ,2) 基于计算复杂性的测度方法 (不完全等同于计算型复杂性, 计算复杂性只是计算 型复杂性的一种）,3) 基于几何学特征的测度方法。 其中计算复杂性的测度属于科尔莫哥洛夫类复杂性 测度, 而其它两类属于非科尔莫哥洛夫类复杂性测 度。

\section{1 基于多样性的群落结构复杂性测度}

\subsection{1 基本定义}

群落结构复杂性与群落多样性有很大的联系 (Kolasa，2005)。在基于多样性的结构复杂性测度 中,一般将群落结构复杂性定义为群落某些结构属 性出现的数量和这些属性的相对多度 (McElhinny et al. 2005)。

\subsection{2 主要测度方法}

基于多样性的群落复杂性测度方法又可分为两
种：一种是将复杂性等同于多样性，直接采用一些生 物多样性的指数来衡量复杂性, 如 Metzger 和 Muller (1996)采用边缘的类型总数和景观中边缘多样性指 数来衡量景观中边缘的复杂性,Hao 等(2002) 采用 群落非相似性指数 ( 1 与 $\beta$ 多样性 SФrensen 指数的 差)来描述植物群落结构的复杂性, 其它还有 Eriksson 等 (2006) 用的生境多样性指数等。另一种是在 多样性定义的基础上, 利用其测度原理, 进行适当的 变换, 来衡量结构复杂性。复杂性毕竟不应完全等 同于多样性, 因此, 后者更具研究价值, 下面以应用 该方法最多的森林结构复杂性为例具体介绍。

由于森林是最复杂的陆地生态系统, 其结构复 杂性的测度研究一方面具有理论价值, 另一方面, 林分中树木的空间布局决定了乌类、昆虫、附生生 物、下层植物及土壤微生物的生境的三维空间” (雷 相东和唐守正, 2002a) “, 空间上异质的森林能容纳 更多的物种，尤其是需要特殊生境的物种” (雷相东 和唐守正 ,2002a）结构的多样性和复杂性对林分的 生物多样性影响很大, 以维护生物多样性为目标的 森林经营必须考虑林分结构的复杂性和多样性并以 其为约束条件 (Gove et al.,1995;Gove,1996)。因 此，结构复杂性指标是森林经营的目标之一，对结构 复杂性的测度研究对于森林生态系统的经营具有应 用指导意义。因此, 该方法在森林结构复杂性研究 中应用最广 ( Pukkala,1997 ;Lahde et al , 1999 ;Zenner 1998, 2000, 2004; Neumann \& Starlinger et al . , 2002; Oliver 2002; ;周涁等 ,2002; 雷相东和唐 守正 2002a 2002b ;Acker et al . , 2003 ;Parkes et al. , 2003 ; 郑景明和罗菊春, 2003)。在此意义上 林分结 构的多样性和复杂性也就十分相似或等同, 以下不 再另行区分。

雷相东和唐守正 (2002a) 对森林结构多样性的 指标进行的综述，从基于多样性的结构复杂性测度 方法的角度看, 这也是对森林结构复杂性测度方法 的一个综述。此后, McElhinny 等(2005) 对此类测度 方法又进行了系统综述, 但没有将上述工作包含在 内。另外, 有关林分结构多样性的一些研究结果, 如 Patil 和 Taillie (1982)、雷相东和唐守正 (2002b) 等也 可纳入本范畴。下面对基于多样性的结构复杂性的 测度方法的介绍就是结合这些研究成果进行的。

根据最后输出形式可以将目前这些方法分为单 数值测度和多值/曲线测度两种。单数值测度用一 个数值来描述复杂性(McElhinny et al .,2005) , 而多 值/曲线测度则用一组有序数据对或一条曲线来描 
述复杂性/多样性 (Patil \& Taillie, 1982 ;雷相东和唐 守正 ,2002b)。

单数值测度目前研究最多、应用最广。这种测 度的构建主要有 3 个环节:1)森林结构属性的选择, 2) 以合适的数学方式将各属性联合起来, 计算出一 个单一数值作为复杂性的测度,3) 各结构属性权重 的确定 (McElhinny et al. ,2005)。在属性选择上 现 有研究往往根据各自的研究目的独立进行, 没有统 一的标准。如 Pukkala (1997) 选择枯倒木和枯立木 的蓄积、阔叶立木蓄积、林分年龄等变量, Lahde 等 (1999)选择各树种的株数分布、胸高断面积、枯立木 蓄积和倒木蓄积、下层植物盖度等作为指标, 郑景明 和罗菊春 (2003) 选择林分各层盖度、站杆、倒木和林 隙的数量等作为林分结构多样性/复杂性的指标, 其 垂直结构指标的计算方法为：

$$
V S I=\sum V S_{i} / n=\sum \sum S C_{i j} S W_{i j} / 8
$$

式中, $V S I$ 样地的垂直盖度指数; $V S_{i}$ :第 $i$ 个小样方 的垂直盖度和; $S C_{i j}$ :第 $i$ 个小样方的第 $j$ 层盖度半 定量值; $S W_{i j}$ : 第 $i$ 个小样方的第 $j$ 层权重。而水平 结构指标的计算方法是 :

$$
H H I=\sum P D_{k} / m \quad P D_{k}=0.5 \sum|a-b|
$$

式中, $H H I$ 样地水平异质性指数; $P D_{k}$ :第 $k$ 对小样 方的相异系数; $m$ 样地内进行相异性比较的小样方 对数; $a, b$ :两个小样方在相同高度层的盖度半定量 值。

在将不同属性合成为一个指数时采用的数学方 式主要有两种：一是加和的方法，如 Lahde 等(1999) 的 LINS 指数，具体计算公式如下：

$$
I N D_{F S}=I N D_{L T}+I N D_{D S T}+I N D_{D F T}+I N D_{C W}+
$$
$I N D_{S P}$

$$
\begin{aligned}
& I N D_{L T}=\sum_{j=1}^{N}\left[\operatorname{LTMAX} X_{j}\left(1-1 / e_{j}^{K K D / 10}\right)\right]\left[\sum_{j=1}^{N}(1-1 /\right. \\
& \left.\left.e^{\left.\left(D 1.3_{j i} / 2\right)^{2} \pi\right) / 100}\right)+\left(1-1 / e^{S N_{j i} / 100}\right)\right] / K_{j} \\
& I N D_{D S T}=\sum_{j=1}^{N}\left[\operatorname{DSTMAX}_{j}\left(1-1 / e^{K K D / 10}\right)\right]\left[\sum_{j=1}^{N}(1-\right. \\
& \left.\left.1 / e^{\left.\left(D 1.3_{j i} / 2\right)^{2} \pi P\right) / 100}\right)+\left(1-1 / e^{S N_{j i} / 100}\right)\right] / K_{j} \\
& I N D_{D F T}=\sum_{j=1}^{N}\left[\operatorname{DFTMAX}_{j}\left(1-1 / e^{K K D / 10}\right)\right]\left[\sum_{j=1}^{N}(1-\right. \\
& \left.\left.1 / e^{\left.\left(D 1.3_{j i} / 2\right)^{2} \pi P\right) / 100}\right)+\left(1-1 / e^{S N_{j i} / 100}\right)\right] / K_{j}
\end{aligned}
$$

式中, $I N D_{F S}$ : 整个林分的复杂性指数; $I N D_{L T}$ 活立木 指数; $I N D_{D S T}$ : 死立木指数; $I N D_{D F T}$ : 倒木指数; $I N D_{C W}$ 烧焦木指数, 为 $0 、 0.5$ 或 $1 ; I N D_{S P}$ 特殊树种 指数, 为 $0 、 0.5$ 或 $1 ; K_{j}$ 胸径径阶数 ; $j$ 树种 $; K D$ :径 阶宽度 $(\mathrm{cm}) ; D 1.3_{j i}$ 树种 $j$ 的第 $i$ 个径阶的平均胸
径 $(\mathrm{cm}) ; S N_{j i}$ 树种 $j$ 的第 $i$ 个径阶的株数; $L T M A X_{j}$ : 树种 $j$ 的多样性的最大值; DSTMAX $X_{j}$ : 树种 $j$ 的死立 木指取最大值, DFTMAX : 树种 $j$ 的倒木指数最大 值 ; $P$ :死树的长度比例。

二是相互作用的方法，如 Jaehne 和 Dohrenbusch (1997)及 Neumann 和 Starlinger(2001) 用乘法形式构 建的林分多样性指数，具体计算公式是：

$$
S D=S_{1} \times S_{2} \times S_{3} \times S_{4}
$$

式中，SD 林分多样性指数; $S_{i}, i=1,2,3,4$ 树种、胸 径、树木空间距离和树冠的变异指数; $S D>9$ : 林分 十分复杂多样, $S D<4$ 林分结构比较单一。

在各属性权重的确定上，有采用专家打分的方 法(Oliver, 2002 ;Parkes et al. , 2003) , 也有采用数学 方法 如周涁等 (2002) 根据多序稳态假说确定权重 的方法。该假说认为, 在外界环境相对稳定的前提 下 森林生态系统的稳定性和有序性随时间的推移 而增加，意味着在生态系统的进化过程中，有序性和 稳定性是一致的, 因此可以根据有序性来研究稳定 性。森林生态系统发育的时间越长 就相对越稳定， 而森林生态系统的稳定性和物种多样性密切相关， 因此, 可以根据有序性来研究多样性和复杂性, 用时 间的某种数学关系计算四者的权重。尽管如此，权 重的确定方法总体上主观多于客观，特别是当要求 所构建的指标能够区分林分不同演替阶段或经营水 平时,不同研究人员确定的指标权重和基准值 (Benchmark) 差别都很大。

多值/曲线测度目前研究较少。Patil 和 Taillie (1982)提出了本质多样性 (Intrinsic diversity)的概念, 并用多样性曲线来比较群落间的多样性。其中一种 为右尾和曲线, 另一种为 $\Delta \beta$ 曲线。雷相东和唐守正 (2002b) 对这两种曲线进行了较详细的介绍, 摘录如 下:

$S$ 为群落的物种丰富度, 定义相对多度向量为 $\pi=\left(\pi_{1}, \pi_{2}, \ldots, \pi_{s}\right), \pi_{i}$ 为 $i$ 物种的相对多度，显然 有:

$$
\sum_{i=1}^{S} \pi_{i}=1
$$

相对多度向量可以用任一指标计算，如个体数 量、盖度或单位面积的生物量 取决于对 物种” 的定 义, 即 $S$ 个类的组成。结构多样性 $(\Delta \mathrm{C})$ 是群落的一 个平均特征, 即平均物种稀有度。每个物种稀有度 的确定将决定其多样性或复杂性指数。Patil 和 Taillie(1982)主要讨论了两种类型的稀有度指标，二 分类型和顺序类型。对于二分类型, $i$ 物种的稀有 
度仅仅决定于它的相对多度 $\pi_{i}$ 。此时稀有度记为 $R$ $\left(\pi_{i}\right)$ 。对于顺序类型，i物种的稀有度仅仅决定于 它的序, 对于有序相对多度向量 $\pi, \pi=\left(\pi_{1}, \cdots\right.$, $\left.\pi_{s}\right)$ ，其中 $\pi_{1} \geqslant \pi_{i} \geqslant \cdots \geqslant \pi_{s}$ 。

右尾和曲线与有序相对多向量 (顺序类型) 相对 应。设 $i$ 物种的稀有度函数定义为：

$R(i)=\left\{\begin{array}{l}1(i>j) \\ 0(i \leqslant j)\end{array}\right.$

对于 $s \geqslant j \geqslant 1$, 平均物种稀有度 (群落多样性) 为:

$$
T_{j}=\sum_{i=j+1}^{S} \pi_{i}^{*}
$$

$j=1,2, \ldots, s-1$

显然, $T_{s}=0, T_{0}=1$ 。上式称为有序相对多度向 量 $\pi$ 的右尾和。对于每个群落, $\left(j, T_{j}\right)$ 构成一条曲 线 称为群落的本质多样性曲线。

群落 $\mathrm{C} 1$ 比群落 $\mathrm{C} 2$ 本质上复杂, 是由于 C2 通过 以下过程产生 C1 :1) 引入一个物种 ;2) 在不改变物 种相对多度排序的情况下，从相对多度高的物种转 化为相对多度低的物种 3)物种的重新标记, 即改变 相对多度向量的组成。对于群落 C1、C2 , 若群落 C1 的本质复杂性曲线始终高于 C2 则群落 C1 本质上 比 C2 复杂性高 若群落 C1 的本质复杂性曲线始终 低于 C2 则群落 C2 本质上比 C1 复杂性高; 若两曲 线相交, 则从本质上说, 这两个群落不能比较。 $\Delta \beta$ 曲线则与无序相对多度向量 (二分类型) 相对应。设 $i$ 物种的稀有度函数定义为 :

$$
R(i, \pi)=\left\{\begin{array}{l}
\frac{1-\pi_{i}^{\beta}}{\beta}, \beta \neq 0 \\
-\log \pi_{i}, \beta=0
\end{array}\right.
$$

同样, 由多样性的定义一平均物种稀有度有：

$$
\Delta \beta=\sum_{i=1}^{S}\left\{\frac{1-\pi_{i}^{\beta}}{\beta}\right\} \pi i=\frac{1-\sum_{i=1}^{S} \pi_{i}^{\beta+1}}{\beta}, \beta \geqslant-1
$$

这样，对于每个群落，( $\beta, \Delta \beta)$ 构成一条曲线, 称 为群落的 $\Delta \beta$ 曲线。每个 $\beta$ 值对应一个多样性指 数, 每条 $\Delta \beta$ 曲线就是一个复杂性指数族。约束条 件 $\beta \geqslant-1$ 能保证 $\Delta \beta$ 曲线与右尾和曲线等效。通 常情况下, 两条 $\Delta \beta$ 曲线在 $\beta>1$ 时相交或多处相交 都是可能的。当 $\beta>1$ 时, $\Delta \beta$ 用处不大, 因为超过这 点时, $\Delta \beta$ 曲线收玫很快, 相交不能分辨, 这种情况 下, 需要数值算法找出两条 $\Delta \beta$ 曲线的相交集。因 此,一般取 $1 \geqslant \beta \geqslant-1$ 。对于群落 C1 和 C2 , 若群落 $\mathrm{C} 1$ 的 $\Delta \beta$ 曲线始终高于 $\mathrm{C} 2$, 则群落 $\mathrm{C} 1$ 本质上比 $\mathrm{C} 2$ 复杂性高, 若二者相交, 则它们在本质上不能比较。
多值/曲线测度与多数的单数值复杂性测度不 同。“如果两个群落存在本质多样性排序, 则不同多 样性指数的排序结果应一致; 但并不是任何两个群 落都可以进行生物多样性的比较, 存在本质上不可 比的情况。因而，从生物多样性的角度看, 不是所有 的群落间都可以比较其好坏。” (雷相东和唐守正， $2002 b$ )。该方法在复杂性和多样性的测度上是观念 的革新(雷相东和唐守正, 2002a, 2002b)。

\section{2 基于计算复杂性的测度方法}

\subsection{1 基本定义}

基于计算复杂性的群落结构复杂性的研究还很 少, 该研究的起源背景是, 复杂性与多样性虽有一定 的联系, 但作为独立的概念, 在测度上不应完全等同 于多样性。许多学者意识到这一点, 指出现有基于 多样性测度的复杂性指数虽然可以提供关于群落复 杂性的某些认识, 但很不充分（Anand \& Orlóci， 1996 ;李镇清, 2000), 因此, 许多学者开始研究其它 测度方法, 其中最重要的就是引入信息学中的测度, 即计算复杂性的测度, 这种复杂性的测度本质上也 是一种科尔莫哥洛夫类复杂性测度。

目前文献中还没有具体给出群落结构复杂性的 计算测度的定义, 可以认为, 群落结构的计算复杂性 的定义是对于群落结构的某一个或若干属性, 能够 描述这些属性分布的最小编码长度或程序长度。

\subsection{2 主要测度方法}

在群落结构计算复杂性测度研究中最重要的一 个工作是 Anand 和 Orlóci(1996)，他们提出用最小剩 余码的平均码长 (Huffman 编码的平均码长) 来作为 群落总复杂性 (Total complexity) 的测度, 用 12 阶 Rényi 熵作为结构随机复杂性 (Disorder complexity) 的 测度, 而最小剩余码的平均码长和 12 阶 Rényi 熵的 差作为结构复杂性 (Structural complexity) 的度量指 标, 具体计算方法是 :

$$
\begin{aligned}
& \Delta(S)=L(S)-H_{a}(S) \\
& L(S)=\sum_{j=1}^{q} p_{j} l_{j} \\
& H_{\alpha}(S)=\frac{1}{1-\alpha} \log _{2} \sum_{j=1}^{q} p_{j}^{\alpha}
\end{aligned}
$$

式中, $S$ : 群落名称; $\Delta(S)$ : 群落 $S$ 的结构复杂性测 度, 比特(bit) :信息单位; $L(S)$ :群落 $S$ 的最小剩余 码的平均码长 (Huffman 编码的平均码长) (bit); $H_{\alpha}$ $(S)$ : 群落 $S$ 的 $\alpha$ 阶 Rényi 熵,一般 $\alpha=12$ (bit); $p_{j}$ : 所选属性 (群落的物种组成等) $j$ 的比例 ; $l_{j}$ : 属性 $j$ 的最小剩余码的码长 (bit)。

最小剩余码 (Huffman 编码)算法为 : 
1)把各成分按概率从大到小排成一列。2)把概 率最小的两个分成一组 : 上面一个编为 0 ,下面一个 编为 1 , 并将这两个符号的概率加起来, 将其结果再 与尚未处理过的符号重新按大小排序。3)重复步骤 2) ,直到所有信源符号都处理完。4) 从右向左依据 编码路径返回 就得到各码字和码长。

Anand 和 Orlóci ( 1996 ) 还对该结构复杂性指标 在不同群落结构中的数值分布情况进行了分析, 他 们测试的群落结构包括两类:一是模拟的群落结构， 包括群落种组成的物种数不同 (5 到 20 个种)、不同 优势度(总个体数分别是 500 和 2000 ,从完全均匀到 优势种的个体数量分别是 495 和 1881) 和不同的分 布格局(单调的、单峰和双峰) :二是对于一些梯度上 的实际群落。他们在 2000 年又对该测度进行了详 细的解释并提供了相关的数学和信息学背景知识 (Anand \& Orlóci 2000) ,同时对在不同等级中的结构 复杂性的分割进行了研究。在此基础上, 李镇清 (2000) 用相似的方法对中国东北样带 (NECT) 的植 物群落复杂性进行了研究。

该方法与 Patil 和 Taillie (1982) 提出的用右尾和 曲线来比较群落间的多样性的方法中的有序相对多 向量(顺序类型) 有一定联系。本质上讲, Anand 和 Orlóci(1996)的方法就是对一个群落的某一特征, 如 相对多度等的顺序排列的一种描述, 而 Patil 和 Taillie(1982)则是用曲线描述的。最小剩余码的平均码 长完全与顺序排列的形状或分布有关。

另一个重要工作是 Lael (2005) 提出了用移动的 三维窗口来衡量种群时空动态的复杂性。其方法 是, 设某个时刻种群的空间格局组成一个 $m \times n$ 的 矩阵, 矩阵中的数据取值为 0 或 $1, t$ 个时刻的格局 矩阵构成了一个 $m \times n \times t$ 的三维数据体。用一个 大小为 $n(n<<\min (m, n))$ 的立方体窗口以一定顺 序遍历该三维数据体, 对于每个位置 $i$, 记落在该立 方体的数据中数值为 1 的数量为 $M_{i}, M_{i} \in\left(0, n^{3}\right)$, 则该格局的时空复杂性可按下式计算 :

$$
S T C=\frac{-\sum_{i=0}^{n^{3}} p_{i} \ln p_{i}}{\ln \left(n^{3}+1\right)_{-}}
$$

式中, $S T C$ :时空复杂性; $p_{i}: M_{i}$ 的比例 $; n$ :窗口大 小。

目前关于生态学中的群落等结构的计算复杂性 的研究工作还很少, 但十分有意义, 至少从概念上使 结构复杂性与生物多样性有了一定的区分, 同时也 指出了一个方向, 即可以用计算复杂性或计算测度
来衡量生态学中的结构复杂性。

\section{3 基于几何学特征的测度方法}

\subsection{1 基本定义}

用群落的一些属性的几何特征, 如分数维等来 表征的群落结构复杂性。

\subsection{2 基本方法}

基于几何学特征的结构/动态复杂性测度主要 采用分数维和其它一些形状指数, 如 van Hees (1994) 用植物群落边缘的分数维作为复杂性的测 度, 研究了美国阿拉斯加的植被复杂性; Marsden 等 (2002) 用分数维、平均斑块面积及其标准差、景观形 状指数等几何指标研究了马来西亚热带森林下层植 被的复杂性; Moser 等(2002) 用斑块的分数维、面积 加权分数维、边缘平均直径面积比、平均形状指数等 描述了澳大利亚乡村景观斑块的复杂性。

Zenner(1998)提出了描述林分结构复杂性的林 分复杂性指数 (Stand complexity index, SCI) , 并用该 指标研究了保留木和老龄木对林分结构复杂性的影 响(Zenner,2000,2004)。该方法基于树木位置, 把各 个水平点的格局 (胸径或树高等)转化成三角面, 然 后将整个林分中由这些三角面构成的三角网的表面 积与其投影面积之比作为 $S C I$ 指标, 具体计算公式 为:

$$
S C I=\frac{S C I^{*}}{A_{T}} \quad S C I^{*}=\sum_{i=1}^{N} 0.5\left|a_{i} \times b_{i}\right|
$$

式中, $S C I^{*}$ : 所有不规则三角网的表面积; $A_{T}$ : 所 有不规则三角网的投影面积; $i=1,2, \ldots, N ; N$ :三 角面的个数(将每株树都看成是三维空间 $(x, y, z)$ 中的一个不规则的空间数据点, 其中 $x 、 y$ 为每株树 的水平位置坐标, $z$ 为每株树的其它属性如树高或 胸径。这样 3 株相邻木在三维空间中组成一个三 角面) ; $a_{i} \times b_{i} \mid$ 为向量 $A B$ 和向量 $A C$ 的积, $A B$ 的 坐标为 $a_{i}=\left(x_{i b}-x_{i a}, y_{i b}-y_{i a}, z_{i b}-z_{i a}\right), A C$ 的坐标 为 $b_{i}=\left(x_{i c}-x_{i a}, y_{i c}-y_{i a}, z_{i c}-z_{i a}\right)$ 。

该方法与前面几种基于几何特征的测度方法不 同，值得关注。

\section{3 现有方法中存在的问题}

\section{1 共性的问题}

可比性问题是现有各种方法中存在的共同问 题。无论是基于多样性的测度还是基于计算复杂性 的测度, 都存在着不同复杂性的结构具有相同指数数 值的问题。该问题涉及到了相应指标的区分能力问 题，作者曾对 Anand 和 Orlóci(1996)提出的 Huffman 编 
码平均码长描述的总复杂性在不同物种丰富度的群 落中的区分能力进行了测试，所得结果表明，该指标 的区分能力不很强。前面介绍的 Patil 和 Taillie (1982) 提出的本质多样性(Intrinsic diversity)本质上不可比的 推断可能会对该问题的解决有所启迪。

\section{2 个性的问题}

1) 对于基于多样性指标的结构复杂性测度指 标 如 McElhinny 等 (2005) 所言, 如何客观地选择结 构属性，如何将这些属性采用合适的数学方式结合 在一起，及如何分配权重都是需要研究的，特别是如 何客观科学地确定各属性的权重是十分困难, 目前 还没有解决好。此外, 由于基于多样性的结构复杂 性指标与多样性指标关系密切, 在复杂性与多样性 之间如何区分也是十分重要的, 否则, 仅将复杂性作 为多样性的另一种说法 就没有太多意义了。

2) 对于结构的计算复杂性测度而言, 有下列 3 个问题 :

a. 复杂性的计算测度既然在概念上与多样性 指数不同, 那么两者之间最好独立或相关很弱。但 Shannon-Wienner 指数作为信息熵, 本身就是编码的 理论上的最短码长 (傅祖芸, 2001) ,与 Huffman 编码 的平均码长自身的相关性很高，据作者的计算机模 拟, 两者的相关系数超过了 0.99 。因此, Huffman 编 码不是群落总复杂性的最好计算测度。

b. 计算测度与现有多样性测度的概念关系还 不十分明确。Anand 和 Orlóci (1996) 提出的总复杂 性、结构复杂性和基于无序的复杂性等概念十分有 启发性, 但由于所用的 Huffman 编码的平均码长和 Shannon-Wienner 指数及 Rényi 摘的高相关性, 该问 题并没有得到很好解决。

c. 计算测度的生态学意义目前还不清楚。这 里主要有以下 3 个层面的问题 : 一是 Anand 和 Orlóci (1996)采用的最小描述码长与 12 阶 Rényi 摘的差作 为结构的测度, 但该测度具体与群落的哪些特征有 关目前还不清楚。二是良好的群落结构复杂性测度 应能够区分不同演替阶段和干扰过程的群落或系 统。在 Anand 和 Orlóci (1996) 及李镇清 (2000) 等的 工作中，虽然将所提出的方法用于在时空和环境梯 度上不同群落的结构复杂性的描述中, 但对其生态 意义或可比性并没有给出明确的回答。L Lael(2005) 的方法只是在模拟的种群中进行了尝试, 缺乏在实
际种群中应用数据的支持。因此，现有这些测度能 否达到上述区分要求还是未知, 还需深入研究。三 是如果生态结构复杂性的计算测度不具有生态意 义，与人们对复杂性的认知不具可比性，那么计算测 度的意义就不大了。这方面还缺乏研究。

\section{4 对末来研究的展望}

如前面所述, 由于方法上的相似性, 群落结构复 杂性的测度方法也可以应用到其它尺度上的生态结 构的复杂性描述中。因此, 群落结构复杂性的测度 问题，因其基础地位而显得十分重要，但由于复杂性 自身定义的多样化, 其测度方法和指标也很多样, 目 前还很难建立统一的多样性测度方法和指标。但无 论怎样, 在未来研究中应对以下三个方面给予高度 重视。

\section{1 生态结构的计算复杂性测度}

计算复杂性很有前景，尽管吴䑣(2004) 所提出 的复杂性概念的本质基础应该是计算复杂性概念的 说法可能不会所有人都同意, 但对于群落等生态结 构的复杂性测度而言, 由于计算型测度具有可操作 性, 无疑应当加强研究，特别是科尔莫哥洛夫类的测 度研究。这是一个新的领域, 核心目标是建立即具 有区分力、又与多样性在概念和数值上都有一定区 别的生态结构计算复杂性的测度方法。要实现该目 标, 比较便捷的途径是将不同的科尔莫哥洛夫类复 杂性测度在生态结构复杂性测度中加以应用。如吴 䑣(2004)所总结的, 目前有数十种复杂性的科尔莫 哥洛夫类的复杂性测度, 在其它一些领域已有成功 的应用范例 ${ }^{1}$ (Bates \& Shepard , 1993 ;李明和 Vitáni， 1998 ; 蔡立羽等,1999; 伦立军等,2004; 佟春生等, 2005）完全可以将它们应用到各尺度的生态结构 (既有模拟的结构, 也可有实际的结构) 复杂性描述 中 然后再从中篮选出合适的测度。

\section{2 结构复杂性测度中的属性权重的科学确定方} 法

该问题在基于生物多样性的结构复杂性测度研 究中可能表现得更突出一些, 事实上, 对于计算复杂 性而言, 也存在着同样的问题。因为结构往往用多 元属性刻画才能更完善, 只要是采用多元属性, 而最 后测度指标又是单一数值时, 必然产生在不同属性 (特征)之间赋予权重的问题。目前虽有一些数学上 
的、相对客观的方法，但产生的权重是否有明显的生 态意义还不明确, 需要进一步研究。

\section{3 生态结构复杂性的测度和生态功能、过程之间} 的联系

MacArthur 和 MacArthur(1961) 提出了用来描述 林分中树叶空间分布状况的叶高多样性指数 (FHD), 同时研究了该指数与林分中鸟类多样性的 关系, 指出两者之间存在着线性关系。该研究将生 态结构复杂性与系统功能或过程联系起来了。目前 这方面的工作还不多, 特别是生态结构计算复杂性 与相应实体的功能、过程的联系还没有。这将是一 个比较有趣的领域, 需进一步加强。

以上问题的解决，将会更好地推动生态复杂性 的研究，加深人们对复杂生态问题的的理解。

\section{参 考 文 献}

Acker SA, Gregory SV, Lienkaemper G, McKee WA, Swanson FJ, Miller SD (2003). Composition, complexity, and tree mortality in riparian forests in the central Western Cascades of Oregon. Forest Ecology and Management, 173, 293 - 308.

Anand M, Orlóci L (1996). Complexity in plant communities: the notion and quantification. Journal of Theoretical Biology, 179, $170-186$.

Anand M, Orlóci L (2000). On hierarchical portioning of an ecological complexity function. Ecological Modelling, 132, $51-62$.

Bascompte J, Solé RV (1995). Rethinking complexity: modeling spatiotemporal dynamics in ecology. Trend of Ecology and Evolution, $10,361-366$.

Bates JE, Shepard HK (1993) . Measureing complexity using information fluctuation. Physics Letter A, 172, 416-425.

Boero F, Belmonte G, Bussotti S, Fanelli G, Fraschetti S, Giangrande A, Gravili C, Guidetti P, Pati A, Piraino S, Rubino F, Saracino OD, Schmich J, Terlizzi A, Geraci S (2004). From biodiversity and ecosystem functioning to the roots of ecological complexity. Ecological Complexity, 1, $101-109$.

Bolliger J, Lischke H, Green DG (2005). Simulating the spatial and temporal dynamics of landscapes using generic and complex models. Ecological Complexity, 2, $107-116$.

Cai LY (蔡立羽), Wang ZZ (王志中), Zhang HH (张海虹) (2000) . Surface EMG signal classification method based on complexity measure. Acta Biophysica Sinica (生物物理学报), 16, 119 - 124. (in Chinese with English abstract)

Dupuy L, Fourcaud T, Stokes A, Danjon F (2005). A densitybased approach for the modeling of root architecture: application to Maritime pine (Pinus pinaster Ait.) root systems. Journal of
Theoretical Biology, 236, 323 - 334 .

Eriksson BK, Rubach A, Hillebrand H (2006) . Biotic habtat complexity controls species diversity and nutrient effect on net biomass production. Ecology, 87, 246-254 .

Fleurant C, Duchesne J, raimbault P (2004) . An allometric model for trees. Journal of Theoretical Biology, 227, 137-147.

Fu ZY（傅祖芸）（2001）. Information Theory-Basic Principle and Application (信息论一一基础原理与应用). Electronics Industry Press, Beijing, 79. (in Chinese)

Gove JH (1996). Optimizing the management of uneven-aged spruce-fir stands while preserving structural diversity. In: Bachmann P, Kohl M, Paivinen R eds. Assessment of Biodiversity for Improved Forest Planning. Kluwer Academic Publishers, Monte Verita, Switzerland, 123 - 134.

Gove JH, Patil GP, Taillie C (1995) . A mathematical programming model for maintaining structural diversity in uneven-aged forest stands with implications to other formulations. Ecological Modelling, 79, $11-19$.

Green DG, Sadedin S (2005). Interactons matter-complexity in landscapes and ecosystems. Ecological Complexity, 2, 117 130 .

Hao BL(郝柏林) (2001). Characterization of complexity and "the science of complexity". Physics (物理), 30, 466 - 471. (in Chinese)

Hao ZQ, Yu DY, Deng HB, Jiang P (2002). Study on complexity of plant communities at different altitudes on the northern slope of Changbai Mountain. Journal of Forestry Research, 13(1), 17 20 .

Hoegh-Guldberg O (2006). Complexities of coral reef recovery. Science, $311,42-43$.

Jaehne S, Dohrenbusch A (1997). Ein Verfahren zur Beurteilung der Bestandesdiversität. Forstwissenschafiliches Centralblata, $116,333-345$.

Jin LW(金吾伦), Guo YL(郭元林) (2004) The science of complexity and their evolution. Complex Systems and Complexity Science (复杂系统与复杂性科学) , 1(1), 1-5. (in Chinese with English abstract)

Keränen SVE (2004). Simulation study on effects of signaling network structure on the developmental increase in complexity. Journal of Theoretical Biology, 231, 3-21.

Kolasa J (2005) . Complexity, system integration, and susceptibility to change: biodiversity connection. Ecological Complexity, 2, $431-442$.

Kondoh M (2003). Foraging adaptation and the relationship between food-web complexity and stability. Science, 299, 1388 1391 . 
Lael P (2005) . Quantifying the complexity of simulated spationtemporal population dynamics. Ecological Complexity, 2, 175 184.

Lahde E, Laiho O, Norokorpi Y, Saksa T (1999). Stand structure as the basis of diversity index. Forest Ecology and Management, $115,213-220$.

Lei XD(雷相东), Tang SZ (唐守正) (2002a). Indicators on structural diversity within stand: a review. Scientia Silvae Sinicae (林业科学), 38, $140-146$. (in Chinese with English abstract)

Lei XD(雷相东), Tang SZ(唐守正) (2002b) . Community intrinsic diversity ordering and its application. Forest Research (林业 科学研究) , 15, 285-290. (in Chinese with English abstract)

Lemper A, Ziv J (1976). On the complexity of finite sequences. IEEE TRANS. IT, 22, $17-93$.

Li M(李明), Vitáni PMB (1998). Description of Complexity (描述 复杂性). Science Press, Beijing, 41. (in Chinese)

LI ZQ (李镇清) (2000). The complexity and diversity of typical plant communities along the Northeast China Transect (NECT). Acta Botanica Sinica (植物学报), 42, 971-978. (in Chinese with English abstract)

Lindsey G (2004). Testing non-equilibrium theories in savannas: 1400 years of vegetation change in Tsavo National Park, Kenya. Ecological Complexity, 1, $281-298$.

Lun LJ(伦立军), Ding XM(丁雪梅), Li YM(李英梅) (2004). A new method about the complexity metrics of program. Application Research of Computers (计算机应用研究), 21 (2), 37 39. (in Chinese with English abstract)

Ma F(马飞), Xu XF(许晓风), Zai BP(翟保平), Cheng XN(程 遐年) (2003). The framework of complexity theory in insect pest disaster research. Entomological Knowledge (昆虫知识), 40, 307 - 312. (in Chinese with English abstract)

MacArthur RH, MacArthur JW (1961). On bird species diversity. Ecology, 42, 594 - 598 .

Marquet PA (2000). Invariants, scaling laws, and ecological complexity. Science, 289, $1487-1488$.

Marsden SJ, Fielding AH, Mead C, Hussin MZ (2002). A technique for measuring the density and complexity of understorey vegetation in tropical forests. Forest Ecology and Management, $165,117-123$.

Maurer BA (2005). Satistical mechanics of complex ecological aggregates. Ecological Complexity, 2, 71-85.

McElhinny C, Gibbons P, Brack C, Bauhus J (2005) . Forest and woodland stand structural complexity: its definition and measurement. Forest Ecology and Management, 218, 1 - 24.

Metzger JP, Muller E (1996) . Charactering the complexity of land- scape boundaries by remote sensing. Landscape Ecology, 11, 65 -77 .

Moser D, Zechmeister HG, Plutzar C, Sauberer N, Wrbka T, Grabherr G (2002). Landscape patch shape complexity as an effective measure for plant species richness in rural landscape. Lanscape Ecology, 17, 657 - 669 .

Neumann M, Starlinger F (2001). The significance of different indices for stand structure and diversity in forests. Forest Ecology and Management, 145, 91 - 106.

Oliver I (2002). An expert panel approach to the assessment of vegetation condition within the context of biodiversity conservation. Ecological Indicators, 2, $223-237$.

Parkes D, Newell G, Cheal D (2003). Assessing the quality of native vegetation: the 'habitat hectares' approach. Ecological Management and Restoration, 4, 29 - 38.

Parrish JK, Edelstein-Keshet L (1999) . Complexity, pattern, and evolutionary trade-offs in animal aggregation. Science, 284, 99 -101 .

Patil GP, Taillie C (1982). Diversity as a concept and its measurement. Jouranl of American Statistical Association, 77, 548 561 .

Pukkala T (1997). Integrating forest-level and compartment-level indices of species diversity with numerical forest planning. Silva Fennica, 31, 417 - 429 .

Rind (1999). Complexity and climate. Science, 284, $105-107$.

Storch D, Gaston KJ (2004). Untangling ecological complexity on different scales of space and time. Basic and Applied Ecology, 5, $389-400$.

Tong DS(佟春生), Huang Q(黄强), Liu H(刘涵), Liu JP(刘俊 萍) (2005). Study on runoff series complexity based on approximate entropy. Journal of Northwest Sci-Tech University of Agriculture and Forestry (Natural Science Edition) (西北农林科技大 学学报 (自然科学版)), 33(6), 121-126. (in Chinese with English abstract)

van Hees WWS (1994). A fractal model of vegetation complexity in Alaska. Landscape Ecology, 9, 271 - 278 .

Vogradov AE, Lynch M, Conery JS (2004). Testing genome complexity. Science, 304, 389-390.

Wang L(王莉), Zhang XY(张晓艳), Zhong Y (钟扬), Nan P(南 蓬) (2002). Advances in biocomplexity studies. Biodiversity Science (生物多样性), 10,238-242. (in Chinese with English abstract)

Wu T(吴䑣) (2004). On concepts of complexities and its meaning in research. Journal of Renmin University of China (中国人民 大学学报), 5, 1-9. (in Chinese with English abstract)

Xue XJ(薛小杰), Tan KL(谭克龙) (2005). The progress of geo- 
scientific study on cellular automata. Coal Geology of China (中 国煤田地质), 17(3), 12-14. (in Chinese with English abstract)

Zenner EK (1998). A New Index for Describing the Structural Complexity of Forest. PhD dissertation, Oregon State University, Corvallis, Oregon, USA.

Zenner EK (2000). Do residual trees increase structural complexity in Pacific Northwest coniferous forests? Ecological Applications, $10,800-810$.

Zenner EK (2004). Does old-growth condition imply high live-tree structural complexity? Forest Ecology and Management, 195, $243-258$.

Zhang ZB (张知涁), Wang ZW (王祖望), Li DM (李典谟) (1998) . Ecological complexity - review and prospect. Acta Ecologica Sinica (生态学报), 18,433-441. (in Chinese with
English abstract)

Zhao WJ(赵文杰), Liu ZL(刘兆理) (2003). The application of cellular automata in environmental science. Journal of Northeast Normal University (Natural Science Edition) (东北师大学报(自 然科学版)), 35(2), 87 - 92. (in Chinese with English abstract)

Zheng JM(郑景明), Luo JQ(罗菊春)（2003）. Structural diversity of broadleaved-Korena pine forest in Changbai Mountain. Biodiversity Science (生物多样性)，11，295 - 302. (in Chinese with English abstract)

Zhou B(周涁), Zheng XX(郑小贤), Zhong Y(钟艳), Lei XD(雷 相东), Li DL (李冬兰) (2002). Synthetic indices for stand species diversity. Journal of Beijing Forestry University (北京林 业大学学报), 24(2), 22-26. (in Chinese with English abstract) 